\title{
Bibliología (ciencia del libro) y ciencias de la documentación
}

\author{
Manuel José Pedraza Gracia \\ Universidad de Zaragoza (España)
}

\section{Resumen}

Se analiza la situación en la que se encuentra la bibliología dentro de los ámbitos en los que se han desarrollado y se desenvuelven las ciencias de la documentación. Para esto se establece, en primer lugar, una aproximación a la evolución conceptual de la bibliología entre los estudiosos y sus estudios relacionándolos con las ciencias de la documentación y estableciendo las diferentes perspectivas existentes. Como resultado del análisis precedente se intenta, en segundo lugar, comprender las mutaciones producidas en el desarrollo de la influencia de las ciencias del libro en la formación de los profesionales de las ciencias de la documentación hasta llegar a la posición actual haciendo especial mención a su situación dentro de las enseñanzas regladas universitarias y a la demanda laboral existente, especialmente en el campo público. Por último, como tercer paso, se analiza dónde y cómo se sitúa la investigación en las ciencias del libro dentro del panorama general de las ciencias de la documentación.

Palabras clave: Bibliología. Bibliografía. Documentación. Historia del libro. Historia de las bibliotecas. Bibliofilia. Ciencias de la documentación.

\section{Abstract}

The status of Bibliology inside the territory of Information Science is analysed. First, the evolution of the concept of Bibliology in relation with the Information Science among its main scholars is analyzed, establishing the different perspectives. Thereafter, the role of the sciences of book in the education of the information professionals up to the present is examined, with an especial emphasis in the official higher education and the existing professional demand, notably in the public field. Finally, the book-related research is contextualized in the general frame of the Library and Information Science.

Keywords: Bibliology. Bibliography. Booklore. Documentation. Books History. Libraries History. Bibliophily. Information Sciences. 


\section{Introducción}

En las últimas dos décadas se han producido una serie de fenómenos - que están todavía por analizar en profundidad - derivados más de la evolución metodológica, especialmente por lo que se refiere a la técnica de los procedimientos, que del desarrollo de la ciencia documental por sí misma. De hecho, los métodos y procedimientos usados en los centros de información con anterioridad a la primera mitad de la década de los ochenta del siglo pasado y los empleados en estos años iniciales del siglo presente no se parecen en absoluto. Es más, han variado los servicios, produciéndose una mayor imbricación de los tipos de centros; también se han modificado las relaciones con los usuarios, perdiéndose en muchos casos el contacto directo; además, se tiende a la externalización de algunas de las actividades técnicas más características; por último, se ha modificado de forma cuantitativa, sin lugar a dudas, la presencia de los diversos tipos documentales - efecto, este, derivado especialmente de la lógica adecuación de las preferencias de los usuarios a las posibilidades tecnológicas, esto es, la adecuación de los centros a la ley de oferta y demanda.

Estas modificaciones, que en ningún caso pueden calificarse como radicales ya que no varía ni el objeto (libro-documento), ni el fin (informar), ni los sujetos (documentalista y usuarios), tienen su reflejo en la situación en la que se encuentran las técnicas, las artes y las ciencias que componen las ciencias de la documentación. Aquí se pretende analizar la situación en la que se encuentra la bibliología y, debido a la estrecha relación que tiene con esta, la bibliografía, ya que se trata de las dos disciplinas que se pueden denominar (o que se han venido denominado) del libro y que constituyeron una parcela muy importante (básica) otrora de las ciencias de la documentación. Entre los contenidos de estas, se van a encontrar, además, otras, especialmente: la historia del libro, la historia de la imprenta, la historia de las bibliotecas, la bibliofilia...

Dentro de los ámbitos en los que se han desarrollado y se desenvuelven los estudios de las ciencias de la documentación, este análisis se centra fundamentalmente en tres líneas principales: la conceptual-estructural, en primer lugar, intentando despejar las situaciones atingentes y contingentes entre estas ciencias y artes; la de su situación en la formación del profesional, relacionada con las necesidades de los mercados laborales; y, por fin, la de su representación dentro de la investigación; en este último caso, mediante una muy somera aproximación cuantitativa que no pretende en ningún caso ser un análisis bibliométrico estricto.

El objetivo principal de este trabajo es, por consiguiente, obtener un panorama general de la ubicación de las disciplinas del libro, en general, y de la bibliología en particular, con respecto a las ciencias de la documentación en la actualidad 
con el fin de obtener, si es posible, una perspectiva aproximada de una ubicación en aquellas y de las causas que la han llevado a esa posición.

\section{Una sucinta aproximación conceptual}

Para llevar a cabo todo lo que se pretende, ya mencionado en los párrafos precedentes, ha de establecerse, en primer lugar, una aproximación, siquiera muy compendiada, a la evolución conceptual de las materias previamente determinadas entre los estudiosos y sus estudios, tanto los de carácter general como en otros más específicos en el entorno cambiante que ha supuesto durante el último cuarto del siglo pasado la (r)evolución de las ciencias de la información y de la documentación, definiendo las diferentes perspectivas existentes. Todo ello, ya sea por lo que hace referencia a la relación entre estas mismas materias (no por evidente necesariamente estática), ya sea, también, por lo que se refiere a su relación con otras materias que en la actualidad tienen límites y contenidos más determinados: bibliografía y documentación.

\subsection{Bibliología}

Según el Diccionario de la Real Academia Española (2004) es el "estudio general del libro en su aspecto histórico y técnico". Entre los estudiosos hay en la actualidad, sin embargo, diversas posturas, como se puede apreciar más adelante.

El primer precedente sobre el uso del término bibliología es el que figura en el Dictionnaire raisonné de Bibliologie de Gabriel Peignot, publicado en 1802 con un suplemento de 1804 que la define, siguiendo el principio etimológico puro, simplemente como "ciencia del libro". Esta amplia definición será seguida durante la centuria por el bibliófilo Octave Uzanne, que la retoma en 1896.

Un seguidor español de esta postura fue el profesor y bibliotecario Baldomero Díez y Lozano, que en 1925 publicó en Murcia un Curso de bibliología en el que comentaba al respecto de la definición de la materia, partiendo de la etimología de la palabra, "que todo lo que se refiere a la entidad del libro constituye la Bibliología” (p. 6).

La bibliología, como ciencia, fue desarrollada, tan solo una decena de años más tarde, por Paul Otlet (1934, p. 9): "Necesitamos ahora no solamente de Bibliografía, descripción de libros, sino de Bibliología, es decir, de una ciencia y una técnica generales del documento"; posteriormente insiste: "No hemos formado aún bien la Bibliología, es decir, una ciencia general que abarque el conjunto sistemático clasificado de los datos relativos a la producción, la conservación, la circulación y la utilización de los escritos y los documentos de toda especie". Y añade a continuación: "Esta ciencia conduciría a [...] reflexionar más profundamente sobre las bases mismas que sirven de fundamento a las disciplinas particulares del libro". Por último, "los esfuerzos deben pues hacerse para constituir

Scire. $11: 1$ (en.-jun. 2005) 27-46. ISSN 1135-3761. 
en ciencia autónoma todos los conocimientos teóricos y prácticos relativos al libro, la Bibliología”. Esto es, Otlet fue el primero en ver la bibliología como ciencia madre, aquella en la que se encuadran todas las otras que la constituyen. En la actualidad, Otlet es considerado como el precursor científico por todos los investigadores y teóricos de la Bibliología, independientemente de la corriente en la que se encuadren.

En momentos más recientes se pueden diferenciar dos corrientes teóricas. En la primera de ellas se encuentran algunos autores que la están definiendo de forma más restrictiva. Así, Jacques Breton (1981, p. 75) entiende que se trata del "estudio sistemático de las condiciones de producción, difusión y utilización de los escritos impresos bajo todas sus formas, incluyendo la búsqueda de los factores de explicación de los fenómenos, tanto en el plano económico y técnico, como político, social y cultural, para descubrir sus perspectivas de evolución tanto cuantitativas como cualitativas". Esta perspectiva restrictiva, ciertamente minoritaria, se centra en la inclusión del término impreso, que eliminaría del objeto de la ciencia todos aquellos documentos que no lo son. La bibliología es, por tanto, para esta corriente la ciencia que se dedica al estudio de los materiales impresos en todos sus aspectos.

La corriente predominante tiene su máxima representación en la Asociación Internacional de Bibliología (AIB). Creada en Túnez en 1988 (Estivals, 1992, p. 40; Diccionario enciclopédico de ciencias de la documentación, 2004, vol. 1 , p. 165, pospone su creación tres años), define la bibliología de forma otletiana como "Ciencia del escrito y de la comunicación escrita" (Estivals, 1992, p. 40). Partidario de esta postura es el autor del único diccionario de bibliología ibérico en tiempos modernos, José Martínez de Sousa, que, aunque en un primer momento la define como "la ciencia que se ocupa del libro en sus aspectos internos y externos, materiales e inmateriales, históricos, terminológicos y técnicos", a renglón seguido añade una definición acorde con la de la AIB: "ciencia que estudia la elaboración, distribución y uso del escrito y de la comunicación escrita" (Martínez de Sousa, 1993, p. 83). Esta corriente amplia del concepto de la bibliología es actualmente la que más seguidores tiene. La diferencia entre ambas ha sido puesta de manifiesto por Martínez de Sousa cuando dice que para la corriente representada por Breton "la bibliología se encarga solamente del estudio del escrito impreso, no del manuscrito" (1991).

Pero estas definiciones no permiten apreciar los elementos que estructuran esa "ciencia del escrito y de la comunicación escrita". La primera aportación a la estructura de la bibliología es la realizada en 1922 por el ruso Nicolay Roubakin, que al introducir el concepto de psicología bibliológica establece una rama de la bibliología completamente nueva. El subtítulo de su obra revela, a la vez, qué entendía el autor por bibliología: "la psicología de la creación de libros, de

Scire. 11 : 1 (en.-jun. 2005) 27-46. ISSN 1135-3761. 
su distribución y circulación, de su utilización por los lectores, las escuelas, las bibliotecas, los libreros, etc.”. El ya mencionado Baldomero Díez dividió tres años más tarde su manual en estos cuatro apartados principales: estudios de erudición, dedicados a los autores; historia general de las bibliotecas; historia de la imprenta; y biblioteconomía.

En la actualidad, el presidente de la AIB, Robert Estivals, en su ponencia "La classification de la bibliologie: pourquoi et comment?", presentada en el IX Coloquio Internacional de Bibliología, celebrado en Túnez en marzo de 1990, estima que la bibliología incluye las siguientes materias (Martínez de Sousa, 1991, p. 45): la diplomática, la gramatología o ciencia de la escritura y del grafismo (manuscritología, paleografía, grafología, semiología tipográfica), la documentología (epigrafía, papirología, codicología, numismática, sigilografía), la editología (bibliografía material y textología), la bibliotecología, la bibliografía científica, la lecturología o ciencia general de la lectura, la bibliometría y las ciencias bibliológicas interdisciplinares (bibliología histórica, sociológica, política, etcétera). Y añade que el sistema general de la comunicación escrita comprende aspectos como las funciones del escrito en la comunicación (interpersonal, colectiva o de masas, literaria y artística, científica y técnica, pedagógica, política y social), el subsistema de producción del escrito (sicología del escritor, lenguajes y escritura, sistema de escritura, sistemas gráficos, soportes del escrito, texto, manuscrito y su reproducción, etcétera), las técnicas de producción y reproducción (técnicas manuales, imprenta, reprografía, técnicas filmicofotográficas, técnicas electrónicas), las categorías de los objetos escritos, las estructuras de edición y de distribución (scriptoria y copistas, imprentas e impresores, edición y editores, industrias de la reproducción, librería, etcétera), la conservación y utilización del escrito: información y lectura (contenido del escrito - la información-), lectura y lectores - bibliofilia -, subsistema de la crítica, subsistema de la conservación (archivos, bibliotecas, mediatecas - biblioteconomía-), el subsistema del escrito no periódico, el subsistema del escrito periódico (periódicos de información general, periódicos especializados), el subsistema del escrito en los productos audiovisuales y el subsistema del escrito electrónico (Martínez de Sousa, 1991).

Estivals avanza más los contenidos de la bibliología, ya que dentro del sistema general de la comunicación escrita incluye el subsistema de los productos auiovisuales.

\subsection{Bibliografía}

Según el Diccionario de la Real Academia, por bibliografía se entiende la "descripción, conocimiento de libros, de sus ediciones, etc." o, en su segunda acepción, "relación o catálogo de libros o escritos referentes a una materia deter- 
minada". Por último, indica que bibliografía textual es el "estudio del proceso material de la fabricación del libro impreso, con vistas a la restitución del texto más fiel a la voluntad del autor" (2004). Desde estas tres acepciones se puede entender la identificación del libro y su descripción, y al resultado de esa descripción se le denomina bibliografía. La tercera de sus acepciones, que la califica como textual, se presenta como el auxiliar del estudio del texto, esto es, la que también se ha denominado con mayor tradición en el mundo literario bibliografía material (Reyes Gómez, 2006).

El bibliotecario Gabriel Naudé publicó en 1633 su Bibliographia politica en Venecia. En esta obra se identifica la bibliografía con una lista de libros. La aportación de Naudé consiste en diferenciar esas listas de libros de las colecciones que poseía una biblioteca, cuyo título incluía las palabras catalogus, bibliotheca, index... (Guerrieri, 1988, p. 130). Esta definición ha venido siendo mantenida desde entonces por numerosos autores europeos que entienden que la bibliografía es la ciencia de los repertorios. Esta corriente se ha denominado "corriente tradicional, enumerativa o continental” (Reyes Gómez, 2006, p. 154).

Desde el primer cuarto de siglo surge dentro del mundo anglosajón la tendencia que entiende que la bibliografía es algo más. Walter Greg realizó una definición magistral en 1932: "lo que al bibliógrafo ocupa es: todo tipo de papel o pergamino con escritura o signos impresos por su forma y no por su contenido ya que esto último no es asunto de él" (p. 247). Se produce, por consiguiente, una divergencia entre los autores del siglo pasado en dos sectores distintos.

Los autores españoles de la última centuria se alinean con la corriente continental de la definición. Javier Lasso de la Vega define Bibliografía como "una sección de la literatura científica cuyo fin es reunir, describir, clasificar y recoger el contenido de los materiales publicados y por publicar" (1975, p. 29). En los momentos más recientes habría que considerar la opinión del autor del último manual español de bibliografía, Juan Delgado Casado, perfecto conocedor desde la perspectiva profesional de la materia. Este autor dice: "Personalmente creo que convendría simplificar un poco la terminología y las acepciones, dejando el término 'bibliografía' para designar la 'lista de libros' hecha con un determinado criterio y para unos determinados fines. Su metodología, aspectos de su elaboración, fases, etc., sería la 'técnica bibliográfica'. El que hace las 'listas', finalmente, sería el "bibliográfo"” (2005, p. 16).

Dentro del mundo académico, con el desarrollo de los centros universitarios de formación en Biblioteconomía y Documentación se mantiene esta forma de entender el concepto. Jaime Moll (1985, p. 145) establece que la bibliografía se ocupa de la

búsqueda, identificación y descripción de manuscritos, impresos y otras publicaciones útiles al conocimiento intelectual. Manuscritos, impresos y otro tipo de publica- 
ciones que denominamos materiales bibliográficos. Estos entran en la categoría de documentos, entendiendo por documento todo objeto portador de información y que sirve para comunicarla. Los materiales bibliográficos, con otros tipos de documentos, son el objeto de la información referente a la misma, por lo que la tarea de facilitarla y la ciencia que se ocupa de la organización de dicha información se conoce, en nuestra área cultural, bajo el nombre de documentación.

Esta misma orientación es hoy la preponderante entre los académicos estudiosos de la bibliografía dentro de las enseñanzas de documentación que siguen la escuela española de la teoría de la documentación creada por José López Yepes. La documentación es definida por López Yepes (1995, p. 321) como “conjunto de disciplinas documentarias que estudian y ejecutan los diversos aspectos del proceso documental (Archivística, Documentación, Museología, Bibliografía, Bibliometría, etc.)". Si bien son muchos los que, además de considerarla incluida en ella, inciden en que es, a la par, el origen de la misma (Moreiro, 1990, p. 45; Cordón y Delgado, 1992, p. 192; Carrizo, 2000, p. 276).

Además, se observa que los autores españoles pertenecientes a la universidad se abonan paulatinamente a la corriente anglosajona que entiende que la bibliografía es, más que el repertorio, la teoría de los repertorios y el estudio de los repertorios, posicionándose en una vía distinta a la de los estudiosos que proceden del mundo profesional, cuyo mayor exponente es Juan Delgado Casado (2005).

Pero, ¿qué constituye la bibliografía, cuáles son las ciencias que pueden encuadrarse dentro de ella?, ¿cuál es su estructura? Al menos dentro de la corriente anglosajona. Según Reyes Gómez (2006, p. 154-171) la bibliografía sistemática, enumerativa y compiladora, que equivale a los postulados de la corriente continental; la bibliografía analítica o crítica, esto es, la ciencia del libro (coincidencia etimológica con bibliología); la bibliografía descriptiva, que se detiene en la búsqueda del ejemplar ideal; la bibliografía textual, que se ocupa de la historia del texto; la bibliografía histórica, que se dedica al estudio de los métodos de producción del libro; y la sociología de los textos. (Hay que tener en cuenta que algunos autores de la corriente anglosajona no entienden que la bibliografía sistemática se incluya en el concepto de bibliografía. Por el contrario, este apartado coincide con el concepto de bibliografía de la corriente continental). Se trata de una estructura amplia que recoge la síntesis de las estructuras propuestas por los autores de la corriente menos restrictiva, sin coincidir exactamente con ninguna de las propuestas individuales. Lo que resalta de esta estructura es que la bibliografía es considerada desde esta corriente como la ciencia del libro.

\subsection{Historia del libro, de la imprenta y de las bibliotecas}

Poco hay que decir sobre la historia del libro y de la imprenta. Esta materia se considera siempre dentro de la estructura de la bibliografía dentro del mundo 
anglosajón (incluida en las mencionadas bibliografía analítica, textual e histórica) e incluso algunos autores la consideran como parte trascendente de la denominada bibliografía material. También se incluye en la bibliología, por definición. Son muy pocos los científicos que dotan de independencia a esta rama de las ciencias del libro a pesar del peso que tiene dentro de la producción científica. Si se tiene en cuenta que los estudiosos de la bibliografía que la consideran como ciencia de los repertorios, suelen ser los que ven a la bibliología como ciencia dentro de la que se halla la historia del libro y de la imprenta y que los que consideran la bibliografía como ciencia del escrito, por tanto incluyendo la historia del libro y de la imprenta, no contemplan la existencia de la bibliología como tal ciencia; la historia del libro y de la imprenta deviene en ambos casos en parte integrante de las que se denominan disciplinas del libro, bien sea incluida en una o en otra.

La historia de las bibliotecas es, en este sentido, algo diferente, ya que la bibliografía no suele incluir esta dentro de su campo de estudio propiamente dicho. Sin embargo, el análisis y estudio de las colecciones es un campo de investigación en auge de la bibliografía actual. Otros aspectos, evolución de las técnicas bibliotecarias, historias de los servicios bibliotecarios, planificación de centros... quedan dentro del ámbito de la biblioteconomía.

La biblioteconomía se considera parte integrante de la bibliología, y lo mismo ocurre con el estudio de las colecciones, por lo que la historia de las bibliotecas forma parte integrante de la bibliología.

\subsection{Bibliofilia}

El Diccionario de la Real Academia (2004) define la bibliofilia de forma etimológica como amor por los libros, especialmente por los raros y curiosos. Quizás este factor de búsqueda de lo más escaso es lo que llama más la atención de la bibliofilia. A este arte se le suele despreciar con cierta ligereza, ya que se entiende que es esta una afición, un capricho de gentes adineradas, en lugar de lo que realmente es, un arte, el de reunir y estudiar una colección de libros y, en ocasiones, documentos relativos a un tema, o a un lugar, o por las características especiales de los ejemplares, no necesariamente antiguos, ni raros ni curiosos. Existen excelentes colecciones de libros modernos, primeras ediciones de autores de la generación del 27, de poesía contemporánea o de temáticas curiosas...

Se trata de una de las disciplinas del libro que primero fueron tratadas. La palabra bibliofilia se encuentra ya en Estrabón y puede considerarse la obra de Richard de Bury escrita en 1344-1345, Philobiblon, como el primer estudio de la misma. En la Edad Moderna, Christ Liberus Saldenus publica en Utrecht en 1681 un tratado denominado Bibliophilia sive De scribendis, legendis et aestimandis libris exercitatio. 
Con la bibliofilia ocurre algo similar que con la historia del libro, de la imprenta y de las bibliotecas. El bibliófilo, que precisa de conocimientos bibliográficos, bibliológicos y de historia del libro y de la imprenta, efectúa sus selecciones de documentos entre los ofertados, todo lo contrario de la disposición de numerario suficiente para abonar aquello que se pide por un capricho, preserva y estudia sus libros, busca y reconoce las características de sus ejemplar... La legislación patrimonial elimina el componente de uso exclusivamente personal de ejemplares extremadamente raros abriendo su utilización al conjunto de la población.

La bibliofilia, por esta causa, tiene un componente bibliográfico importantísimo, tanto en su faceta de selección como en la de estudio. Tampoco cabe duda de que la bibliofilia es estructuralmente un arte integrante de la bibliología.

Son muy pocos los tratados españoles dedicados a la bibliofilia (Mendoza, 2002, 2004), que, además, no se centran en encontrar una definición de su objeto; por esta causa, parece que una definición apropiada de bibliofilia es: arte bibliológico, mejor documental, que consiste en reunir y estudiar un conjunto documental armónico para uso personal.

\subsection{Bibliología y bibliografía}

De lo visto hasta este momento puede desprenderse que, si se toman las estructuras de ambas ciencias principales en su formulación más amplia de entre las propuestas por sus teóricos, resulta evidente que ambas engloban una serie de componentes muy similares y aún idénticos.

En efecto, si se analizan las estructuras de ambas se aprecian coincidencias muy notables. Para ello se pueden tomar las excelentes arquitecturas estructurales, mencionadas más arriba, ofrecidas por Robert Estivals (1992) y la AIB para la bibliología, y el esquema (excelente síntesis) realizado por Fermín de los Reyes (2006) tras el estudio de las posiciones de los principales representantes de la corriente anglosajona de la bibliografía.

Tomando como punto de partida las posturas de Estivals, se pueden encontrar, sin profundizar mucho, las siguientes y evidentes relaciones:

1. Dentro de la gramatología incluye la manuscritología y la semiología tipográfica, que, entre otros, son aspectos que estudian la bibliografía histórica y la sociología de los textos.

2. Dentro de la documentología se incluye la codicología, que, como en el caso anterior, es un aspecto de varios de los componentes de la bibliografía y de la sociología de los textos.

3. La editología incluye la bibliografía material, denominación general que equivale a la postura íntegra de diversos autores anglosajones. También

Scire. $11: 1$ (en.-jun. 2005) 27-46. ISSN 1135-3761. 
incorpora la textología, que se identifica con la bibliografía textual y descriptiva.

4. Entre los componentes de la bibliología figura la bibliografía científica, que vendría a ser lo mismo que la bibliografía sistemática.

5. Las ciencias bibliológicas interdisciplinares (bibliología histórica, sociológica, política, etcétera), que son analizadas desde las perspectivas de las bibliografías analítica, textual, histórica o, como es evidente, por la sociología de los textos.

Quedan exentas de forma íntegra cuatro ciencias: la diplomática, considerada como la sigilografía, la paleografía, la cronología y la numismática como ciencias auxiliares de la archivística y de la bibliografía; la bibliotecología; la lecturología, se encuentra, en estos momentos encuadrados dentro de la sociología y de la literatura, pero aspectos como la sociología de la lectura o la legibilidad tiene relación con la biblioteconomía y la bibliografía; y la bibliometría, aunque el origen bibliográfico de esta está fuera de toda duda (Hulme, 1923, como precedente de Otlet, y Pritchard, 1969).

La mayoría de los autores que ponen en relación ambas ciencias siempre incluyen la bibliografía dentro de la bibliología y nunca a la inversa, y resulta evidente que, a tenor de lo defendido por Estivals, la bibliografía en su vertiente más generalista forma parte de la bibliología, como ocurre con otras ciencias también incluidas dentro de las ciencias de la documentación (bibliotecología, bibliometría...) y otras referidas al mundo del escrito. Esta posición ha creado escuela en la esfera internacional de los estudios de las ciencias del libro. Seguramente ambas ciencias, o si se quiere, la ciencia del libro tropieza, como tropiezan otras dentro del mundo de la documentación, con problemas de carácter terminológico. A. M. Lewin Robinson, una vez definida la bibliografía analítica (1992, p. 12), añade: "El resto se describe como ciencias del libro, artes del libro (alemán Bücherkünde) o incluso por esa horrible palabra, 'Bibliología', ya que en

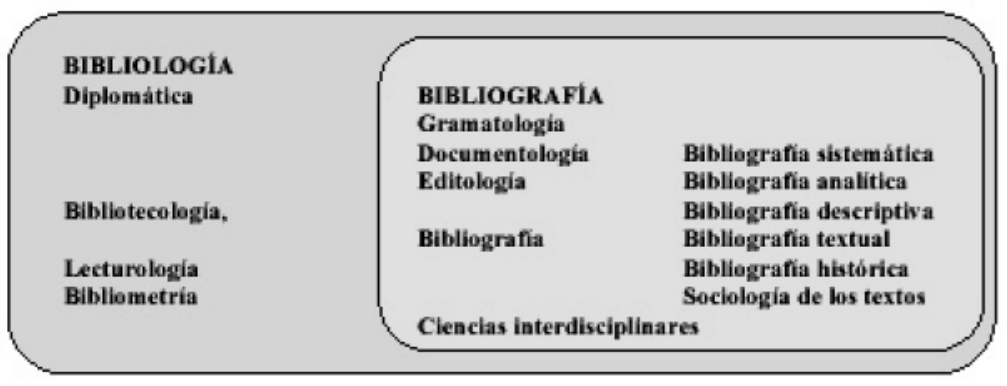

Figura 1. Las ciencias del libro

Scire. $11: 1$ (en.-jun. 2005) 27-46. ISSN 1135-3761. 
inglés tiene preponderantemente, aunque no siempre, una significación religiosa que está entrando con fuerza en algunos países iberoamericanos".

\subsection{Bibliología y documentación}

Es perfectamente conocido que el concepto documentación se entiende bien como "ciencias de la documentación" o bien como "una de las ciencias de la documentación" (Diccionario enciclopédico de ciencias de la documentación, 2004, pp. 454-455). Al objeto de este trabajo interesa esta segunda acepción, ya que, de lo que se trata es de discernir la relación que la bibliología tiene con las componentes de las ciencias de la documentación o si se trata de una ciencia límite con otras materias, como las ciencias y técnicas historiográficas, la literatura, la sociología...

La primera relación remarcable hay que buscarla en el Traité de Otlet. Este no solamente reestructura y desarrolla la bibliología, como ya se ha mencionado previamente, sino que es también el origen de la documentación. En su primer capítulo introduce ambos términos. Se titula "Bibliología o documentología" y aclara: "Ciencias del libro y de la documentación" (Otlet, 1934, pp. 9 y ss.). Otlet es el primero que pone esta relación en evidencia estableciendo una correspondencia entre ellas, que se deriva exclusivamente de la restricción del radical biblion, como pone de manifiesto en los apartados "Partes de las ciencias bibliológicas" y "Terminología y nomenclatura" (Otlet, 1934, p. 18-19). Otlet, como resulta evidente, identifica bibliología y documentología, y a esa ciencia la define como ciencia del libro y de la documentación. Pero, como ya se ha puesto de manifiesto, es importante recalcar que, además de la bibliografía, entre las ciencias, técnicas y artes bibliológicas se han encontrado otras que se hallan plenamente integradas en las ciencias de la documentación siendo consideradas como autónomas. Este es el caso de la biblioteconomía o de la bibliometría. La diplomática, como la paleografía, son consideradas como ciencias auxiliares de la archivística y la codicología, y la lecturología se considera auxiliar de la biblioteconomía y la bibliografía. De todo esto se deduce que la bibliología, si no se puede identificar con documentación a la manera otletiana, cuando menos forma parte integrante de las ciencias de la documentación.

La estructura clásica de las ciencias de la documentación ha sido expuesta en numerosas ocasiones, la última (Diccionario enciclopédico de ciencias de la documentación, 2004) dice al respecto: "también denominadas ciencias del documento, ciencias de la información documental, etc. Tienen por objeto de estudio aquellas disciplinas relacionadas con el documento, esto es, con alguno de los tipos de proceso informativo-documental como la Archivística, Bibliografía, Biblioteconomía, Documentación, Museología, etc.”. Se expresa, en primer lugar, la identificación con "ciencias del documento" o "disciplinas relacionadas

Scire. 11 : 1 (en.-jun. 2005) 27-46. ISSN 1135-3761. 
con el documento", definición esta que recoge el pensamiento otletiano en su forma más literal. Cuando se trata de la estructura de esta ciencia se mencionan la archivística, la bibliografía, la biblioteconomía, la documentación y la museología. En efecto, la biblioteconomía, la bibliografía, la documentación y la archivística, en diverso grado, son consideradas integrantes de la bibliología, ya que son "ciencias del escrito y de la comunicación escrita". En tanto la bibliología entiende por escrito cualquier soporte que contiene información, ha de incluirse también la Museología. De todo esto se deduce que conceptualmente las ciencias del libro (bibliología), como mínimo, son parte integrante de las ciencias de la documentación y en tanto se identifiquen los conceptos de escrito y documento utilizados por los teóricos de ambas disciplinas pueden llegar a comprender objetos plenamente comunes, y a identificarse entre ellas (figura 2).

La diferencia esencial entre ambas se presenta en el objeto. Como se ha visto más arriba, la bibliología tiene por objeto el estudio del documento (del escrito) y su comunicación; por su parte, además, las ciencias de la documentación tienen por objeto el estudio de "aquellas disciplinas relacionadas con el documento, esto es, con alguno de los tipos de proceso informativo-documental" (Diccionario enciclopédico de ciencias de la documentación, 2004). La bibliología se centra especialmente en el objeto desde su producción y desde su recepción, mientras que las ciencias de la documentación se centran, además, en el proceso.

\section{Las enseñanzas de las ciencias del libro}

Como resultado evidente del análisis precedente se intenta, en segundo lugar, comprender las mutaciones producidas mediante el correspondiente estudio diacrónico del desarrollo de la influencia de las ciencias del libro en la formación de

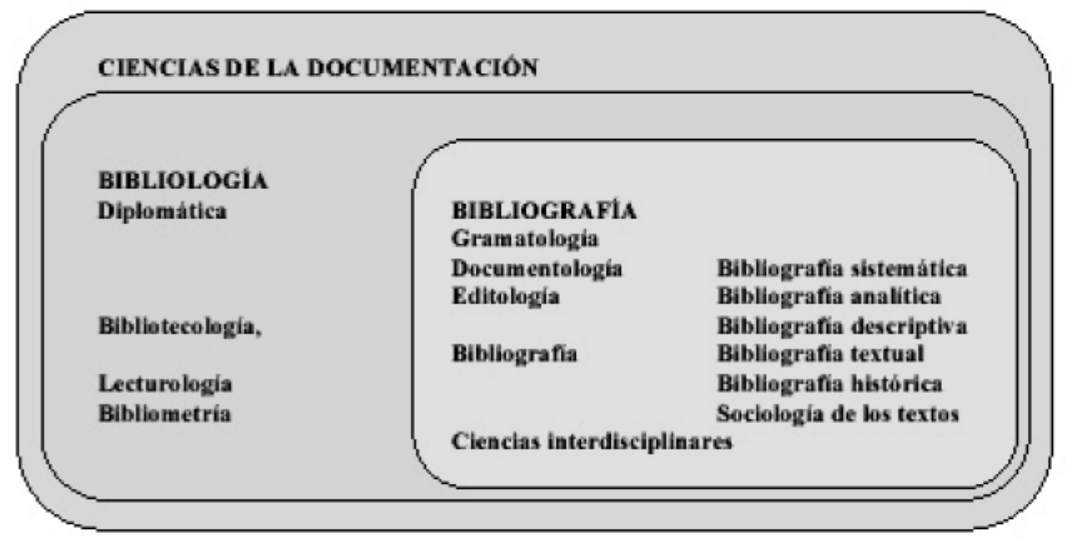

Figura 2. Las ciencias del libro en las ciencias de la documentación

Scire. $11: 1$ (en.-jun. 2005) 27-46. ISSN 1135-3761. 
los profesionales de las ciencias de la documentación hasta llegar a la posición actual, haciendo especial mención a su situación dentro de las enseñanzas regladas universitarias y a la demanda laboral pública existente, para la que se precisan conocimientos especializados en las materias mencionadas.

Aunque existen algunos precedentes de escaso éxito, las enseñanzas de bibliografía y bibliología tienen una estrechísima relación con la aparición de la formación profesional de archiveros y bibliotecarios dentro de la Escuela Superior de Diplomática. En ella, la Bibliografía, primero, y, después, la Bibliología se fueron constituyendo en asignaturas de importancia dentro de la formación de estos profesionales. La Bibliografía mantuvo siempre cierta relación con los estudios de historia literaria dando lugar, en ocasiones, a asignaturas que reunían ambas materias. Por su parte, la Bibliología, cuando se rodeaba de otras materias, se presentaba en relación con asignaturas de Biblioteconomía. Con la desaparición de la Escuela sus estudios pasaron a la Universidad Complutense. Se crearon cátedras que se denominaron Bibliografía (Universidad Complutense) o Bibliología (Universidades de Barcelona y Salamanca). Con el paso del tiempo parte de estos estudios, los de Bibliografía, concluyeron en los de Filología (Universidad Complutense), y los de Bibliología en los de Paleografía (Universidades de Barcelona, donde desaparecen muy pronto, y Zaragoza, donde permanecen en la actualidad, adscritos al Departamento de Historia Medieval, Ciencias y Técnicas Historiográficas y Estudios Árabes e Islámicos) (Reyes Gómez, 2004).

La Escuela de Bibliotecarias de Barcelona solicitó en los años setenta del siglo pasado, en dos ocasiones, su transformación en Escuela Universitaria de Bibliología adscrita a la Universidad de Barcelona (Rodríguez Parada, 2002).

En la década siguiente se crean las enseñanzas de Biblioteconomía y Documentación como estudios oficiales mediante la "Orden de 24 de febrero de 1981, por la que se fijan las directrices de los planes de estudio de las Escuelas Universitarias de Biblioteconomía y Documentación”. En el plan de estudios pertinente se introducen dos asignaturas que se denominaban Bibliografía, la primera introducía entre paréntesis la especificación "introducción a las fuentes de información" y la segunda añadía "Bibliografía general y especializada". Este plan de estudios de 1981 reforzaba la concepción de la bibliografía como "ciencia de los repertorios", entendía la bibliografía como auxiliar de la biblioteconomía por lo que respecta a la referencia. En ningún caso aplicaba la concepción amplia de la bibliografía, hoy vigente. La orden mencionada fue derogada por el Real Decreto 1497/1987 y sustituida por el "Real Decreto 1422/1991, de 30 de agosto, por el que se establece el título universitario oficial de diplomado en Biblioteconomía y Documentación y las directrices generales propias de los planes de estudios conducentes a la obtención de aquel". Entre las materias componentes de estos estudios se introduce "Bibliografía y fuentes de información". La participación de la

Scire. $11: 1$ (en.-jun. 2005) 27-46. ISSN 1135-3761. 
bibliografía, la historia del documento, la historia de las bibliotecas, las fuentes de información, en suma, las asignaturas que originalmente se pueden considerar como parte integrante de las ciencias del libro en los estudios, ronda desde el diez hasta casi el veinte por ciento de la troncalidad y obligatoriedad de los estudios. Por ejemplo, 19 créditos totales en la Universidad Carlos III de Madrid y 19,5 en la Complutense son los casos en los que tienen más peso. Varía también en torno a estos porcentajes la participación de asignaturas optativas ofertadas.

Posteriormente, se publica el "Real Decreto 912/1992, de 17 de julio, por el que se establece el título universitario oficial de Licenciado en Documentación y la aprobación de las directrices generales propias de los planes de estudio conducentes a la obtención de aquel". Por lo que se refiere a la licenciatura en Documentación, las materias relacionadas con estas disciplinas desaparecen de la troncalidad y obligatoriedad y solo en las asignaturas optativas se hallan asignaturas como "Recursos y servicios de referencia", "Historia del libro", "Historia de la bibliografía española", "Fuentes de información en...", "Literatura gris"... Se centran esencialmente en la profundización sobre la historia del documento y en las fuentes de información, generalmente de forma estructurada.

Por su parte, la bibliología prácticamente ha desaparecido de la universidad española; solamente queda una asignatura optativa en los estudios de Historia en la Facultad de Filosofía y Letras de la Universidad de Zaragoza que está siendo impartida por el Departamento de Ciencias y Técnicas Historiográficas y Estudios Árabes e Islámicos, con los siguientes objetivos: “Acceso al conocimiento científico del instrumento de la memoria llamado 'libro', diacrónica y analíticamente considerado (soportes, grafías, ilustraciones, encuadernaciones, depósitos, usos, etc.). Capacitación técnica en orden a la realización de algunas funciones competentes al área libresca"). Dentro del programa de teoría se encuentran "59 lecciones sobre el perfil histórico del libro (manuscrito e impreso) y aspectos de su utilización: descripción bibliográfica, catalogación, análisis de contenido y referencias a la bibliotecología y archivística". Como ejercicios prácticos figuran la "elaboración de cédulas bibliográficas, índices, repertorios, estudio de materiales impresos y manuscritos, test de lecturas" (Bibliología, 2005).

Finalmente, las últimas actuaciones referidas a las enseñanzas oficiales en Información y Documentación (Título de grado en Información y Documentación, 2004) en su propuesta de contenidos comunes y obligatorios (troncalidad) introduce dos materias: (1) "Documentos, unidades y sistemas de información”, en los que se encuentran los contenidos naturaleza de la información, concepto y evolución de los documentos, naturaleza y evolución de los archivos, las bibliotecas y los centros y servicios de documentación, desarrollo histórico y estado actual de la disciplina, legislación y políticas nacionales e internacionales, aspectos culturales, sociales, económicos y legales de la producción y uso de la información 
documental, deontología y perfil del profesional de la información y documentación; y (2) "Fuentes de información", entre cuyas materias se encuentran naturaleza y tipología de las fuentes de información, acceso y consulta de fuentes de información electrónica, análisis y evaluación de fuentes, producción, normalización y difusión de fuentes. Es evidente que los redactores del proyecto han tenido muy en cuenta determinados contenidos relacionados con las ciencias del libro, pues se pone especial intención en los contenidos diacrónicos y en las fuentes de la información documental, si bien se han perdido las denominaciones bibliografía, bibliología, historia del documento... dentro de los contenidos; mientras que, a la vez, se refuerza el término y el concepto de fuentes de información.

Este compendio de aportaciones de las universidades españolas tiene en cuenta la situación de la profesión en Europa y España a la hora de presentar las materias que se precisan en la formación profesional. No parece que hayan tenido el peso suficiente, sin embargo, los aspectos formativos que se exigen en el acceso a la profesión dentro de la función pública. De hecho, si se analiza la última convocatoria de las últimas oposiciones convocadas para el Cuerpo Facultativo en su sección de bibliotecas se apreciará que incluye 43 temas sobre bibliografía, fuentes, historia del libro y de las bibliotecas y patrimonio bibliográfico de un total de 125 temas divididos en cinco grupos. Esto es, algo más de un tercio de la formación teórica exigida tiene que ver con las tradicionales ciencias del libro en las oposiciones para bibliotecario. Pero es que en el primer ejercicio es obligatorio que uno de los tres temas pertenezca al apartado de bibliografía y documentación $\mathrm{y}$ otro al de historia del libro y de las bibliotecas, y en el cuarto es obligatorio que un ejercicio práctico esté relacionado con los temas que constituyen el apartado de bibliografía y documentación. Además, en las oposiciones de archivero, hay una sección entera de 24 temas dedicada a bibliografía y fuentes especializadas en instituciones y archivos.

\section{La investigación en las ciencias del libro}

Por último, como tercer paso consecuente, habrá de analizarse dónde y cómo se sitúa la investigación en las ciencias del libro dentro del panorama general de las ciencias de la documentación.

En muchos lugares se mantienen las asociaciones, como la ya mencionada internacional AIB, y la preocupación por el estudio de la bibliología, como es el caso del Instituto de Investigaciones Bibliográficas mexicano, que se plantea como misión principal realizar e impulsar la generación de conocimientos en los ámbitos de la bibliografía, la hemerografía, la bibliotecología, la bibliología, los archivos y manuscritos y el estudio de fuentes (fuentes que custodian la Biblioteca y la Hemeroteca nacionales de México), con el fin de precisar los campos generales del conocimiento y facilitar o aun permitir su estudio. En España se constituyó,

Scire. $11: 1$ (en.-jun. 2005) 27-46. ISSN 1135-3761. 
el 12 de diciembre de 1990, en la Escuela Universitaria de Biblioteconomía y Documentación de la Universidad de Salamanca, en presencia del profesor Robert Estivals, una comisión gestora del grupo español de la AIB que ha dado lugar a la Asociación Española de Bibliología en 1996. Esta fundó una revista electrónica, Revista española de Bibliología, de la que solamente se ha publicado un número de 1997 (http://www.uv.es/ barrueco/reb/esp/). Además, existe la Asociación Española de Bibliografía, que publica sus Trabajos de la Asociación Española de Bibliografía. De esta publicación han aparecido hasta la fecha dos volúmenes, uno en 1993, editado por la Biblioteca Nacional, y otros dos en 1998 y 2004, editados también por la propia Asociación.

Entre las revistas científicas especializadas en ciencias de la documentación de carácter general se han seleccionado diez, cinco que emanan de las universidades españolas y cinco de entre las de ámbito profesional, si bien, en este caso, se incluyen las publicaciones de algunas asociaciones de ámbito exclusivamente bibliotecario y otras que se dirigen específicamente a profesionales. En los últimos cinco últimos números aparecidos de Anales de Documentación, revista publicada por la Universidad de Murcia desde 1998, disponible en línea (http://www. um.es/fccd/anales/) se han publicado 81 trabajos de los que 6 tienen relación con la bibliografía, la bibliología, la historia del libro... La Revista General de Información y Documentación, editada desde 1992 por la Escuela Universitaria de Biblioteconomía y Documentación de la Universidad Complutense, recientemente convertida en Facultad, ha publicado desde 2001 un total de 89 trabajos de los que 13 pueden incluirse en las que se han denominado ciencias del libro. BID: Textos Universitaris de Biblioteconomia i Documentació, de la Facultat de Biblioteconomia i Documentació la Universitat de Barcelona desde 1998, ha publicado en sus últimos cinco años 62 artículos de los que 5 caben dentro de las ciencias que se estudian en este trabajo. Documentación de las Ciencias de la Información, revista editada por el Departamento de Biblioteconomía y Documentación de la Universidad Complutense desde 1976, ha publicado en el mismo período 86 artículos de los que 6 pertenecen a las ciencias estudiadas. Scire: Representación y Organización del Conocimiento, revista editada en la Universidad de Zaragoza por el profesor García Marco desde 1995 ha publicado en su último quinquenio 109 trabajos de los que 8 se pueden encuadrar en la bibliología o la bibliografía. En el Boletín de la Anabad, publicado desde 1978 por la Confederación de Asociaciones de Archiveros, Bibliotecarios, Museólogos y Documentalistas, heredera del Boletín de la Anaba, han aparecido (una vez eliminados los números monográficos) en los últimos cinco años 87 artículos de los que 7 entrarían dentro del ámbito del campo de estudio. El Boletín de la Asociación Andaluza de Bibliotecarios, editado desde 1984 y disponible en línea (http:. //www.aab.es), ha publicado en el mismo período de cinco años 79 traba- 
jos de los que 10 pueden encuadrarse dentro de las disciplinas del libro. Item: Revista de Biblioteconomia i Documentació, editada por el Col-legi Oficial de Bibliotecaris-Documentalistes de Catalunya desde 1987, ha publicado 77 artículos, de los que solo 4 entrarían en el campo de estudio de este trabajo. El Boletín de la Asociación Asturiana de Bibliotecarios, Archiveros, Documentalistas y Museólogos AABADOM, editado desde 1990, ha publicado en los últimos cinco años 68 trabajos, de los que 9 pueden ser considerados como bibliológicos o bibliográficos. El Profesional de la Información, que se publica desde 1998 y está disponible en línea (http://www.doc6.es/iwe/), ha publicado 105 "artículos", de los que solo uno puede encuadrarse en el mundo de las ciencias del libro.

La presencia de la investigación dentro de estas revistas universitarias es más bien escasa, lo que no sería más que un reflejo de la presencia de estas materias en el ámbito de la formación. La misma tendencia incide en las revistas que se han denominado aquí del ámbito profesional. Este fenómeno no es extraño ya que con la incorporación de los nuevos profesionales de la información y documentación al mercado de trabajo las revistas relacionadas con este campo en España han ido derruyendo los muros que las separaban. El resultado global sería, por tanto: de 843 trabajos publicados 69 pueden ser encuadrados dentro de las disciplinas del libro.

Por lo que se refiere a las reuniones científicas, es suficiente indicar que de las últimas ediciones de tres de las reuniones científicas (elegidas al azar) de carácter general más importantes - Las Jornadas Españolas de Documentación, Ibersid y las Jornades Catalanes d'Informació y Documentació- solamente en Ibersid se tocan temas relacionados con las ciencias del libro.

Pero esto no quiere decir que la bibliografía y la bibliología no tengan interés en España, dentro del ámbito científico. Es más, posiblemente se trate de una materia en auge. Una búsqueda sencilla, por ejemplo en Dialnet, bajo el epígrafe "información y documentación" produce como resultado 77 títulos de publicaciones periódicas, entre las que se encuentran, además de las utilizadas y mencionadas anteriormente, títulos como Pliegos de Bibliofilia (de origen privado y creada en 1998), Signo (editada por el Departamento de Historia y Filosofía de la Universidad de Alcalá de Henares desde 1994 hasta 2004 y continuada por Cultura Escrita y Sociedad), Syntagma: Revista del Instituto de Historia del Libro y de la Lectura (editada desde 2002 dentro de la Universidad salmantina), Pecia Complutense: Revista de la Biblioteca Histórica de la Universidad Complutense, editada desde 2004 por la Biblioteca Histórica Marqués de Valdecilla), Cartas diferentes (publicada de forma privada en La Palma desde 2005), Hibris: Revista de Bibliofilia (publicada de forma privada en Alcoy desde 2001), Historia y Comunicación Social (editada por el Departamento de Historia y Comunicación Social de la Universidad Complutense desde 1996),

Scire. $11: 1$ (en.-jun. 2005) 27-46. ISSN 1135-3761. 
y con poco esfuerzo adicional encontramos Litterae: Cuadernos Sobre Cultura Escrita (publicada por el Instituto de Cultura y Tecnología de la Universidad Carlos III desde 2001) o Avisos: Noticias de la Real Biblioteca (publicada desde 1995). Esto es, una decena de publicaciones periódicas relacionadas con la historia del libro, la bibliología, la bibliofilia y la bibliografía... en curso durante los últimos cinco años, de las que seis surgen precisamente en este período. Si se analizan en profundidad estos datos encontramos que ninguna de estas publicaciones especializadas está sustentada por departamentos de ciencias de la documentación de la universidad española. Las hay privadas y publicadas por departamentos de Historia, Literatura o Ciencias y Técnicas o instituciones relacionadas con estos; y, también, dependientes del mundo profesional de las bibliotecas.

\section{Conclusiones}

La bibliología, según las propias definiciones, origen y estructura concebidas y determinadas por sus principales estudiosos, no puede entenderse en la actualidad alejada de las ciencias de la documentación.

Se detecta un desajuste entre las demandas de conocimientos del mercado laboral en la administración pública y la formación de profesionales en lo referente a las ciencias del libro. No obstante, falta determinar dónde se encuentra el desfase entre un mundo y otro. Quizás este desfase no sea tan evidente en las demandas de conocimientos del mercado laboral en el mercado libre, o quizás directamente no exista, ya que en los nuevos planes de estudios elaborados por el mundo académico se ha tenido en cuenta.

Gran parte de los trabajos con contenidos de historia del libro, de la imprenta y de las bibliotecas, bibliológicos y aun bibliográficos quedan en el margen de las ciencias de la documentación, cuando se analizan desde la perspectiva de la producción científica, aunque sea de forma muy somera, en publicaciones seriadas y en las reuniones científicas.

Los espacios científicos de las disciplinas del libro que no están siendo trabajados por los científicos del mundo de la documentación han sido absorbidos desde el mundo de la historia, la filología, la historia de la ciencia y las ciencias y técnicas historiográficas, todas ellas áreas de conocimientos mucho más potentes que la de las ciencias de la documentación. Es de interés remarcar que los investigadores en estas materias, externos a la formación de documentalistas, no suelen publicar en revistas de documentación.

En consecuencia, en España parece que existe una parcela de las ciencias de la documentación, ya reconocida y desarrollada por Otlet, que se ha difuminado entre otras ciencias, seguramente debido a su carácter interdisciplinar. 


\section{Referencias}

Baldacchini, Lorenzo (1992). Lineamenti di bibliologia. Roma: La Nuova Italia scientifica, 1992.

Bibliología (2005). Zaragoza: Universidad de Zaragoza, Facultad de Filosofía y Letras, 2005. URL: <http:. //fyl.unizar.es/Medieval/Bibliología.html>. [Consulta: sept. 2006]

Breton, Jacques (1981). Bibliologie, communicologie et bricolage idéologique. // Schéma et schématisation, 19 (1981).

Carrizo Sainero, Gloria (2000). Bibliografía. // Manual de fuentes de información. 2a ed. Zaragoza: Confederación Española de Gremios y Asociaciones de Libreros, 2000.

Cordón García, José Antonio; Delgado López-Cózar, Emilio (1992). Bibliografía y ciencias de la información. // Miscelánea-homenaje a Luis García Ejarque. Madrid: FESABID, 1992.

Delgado Casado, Juan (2005). Introducción a la bibliografía. Madrid: Arco/Libros, 2005.

Diccionario enciclopédico de ciencias de la documentación (2004). Madrid: Síntesis, 2004. 2 vols.

Diccionario de la lengua española (2004). 22ª ed. Madrid: Real Academia Española, 2004. URL: <http: //buscon.rae.es/draeI/ >. [Consulta: sep. 2006].

Díez y Lozano, Baldomero (1925), Curso de bibliología. Murcia: Imp. Lourdes, 1925.

Estivals, Robert (1987). La bibliologie, París: Presses Universitaires de France, 1987.

Estivals, Robert (1992). Historia de la bibliología. // Item, 11 (1992), 25-41. URL: <http://www.cobdc. org/cgi-bin/intranet/pdf.pl ?page=item/num11/restivals.pdf]>. [Consulta: sept. 2006]

Greg, Walter (1932). Bibliography - an Apologia. // Collected Papers, editado por J. C. Maxwell (Oxford: Clarendon Press, 1966); publicado originalmente en: The Library, 4th series, 13 (1932), 113-43.

Guerrieri, Guerriera (1988). Nuove linee di biblioteconomia e bibliografía. $3^{\text {a }}$ edizione riveduta, aggiornata e ampliata di Giuseppe di Nitto. Roma: Guida, 1988.

Hulme, E. W. (1923). Statistical Bibliography in Relation to the Growth of Modern Civilization. London: Grafton, 1923.

Lasso de la Vega, Javier (1975). El trabajo intelectual: normas, técnicas y ejercicios de documentación, Madrid: Paraninfo, 1975.

López Yepes, José (1995). La documentación como disciplina: teoría e historia. $2^{\mathrm{a}}$ ed. act. y amp. Pamplona: Eunsa, 1995.

Martínez de Sousa, José (1991). La bibliología, una nueva ciencia. // Asterisco cultural. 4 (1991). URL: <http:. //www.fundacionyuste.org/acciones/aeb/ques.htm>. [Consulta: sept. 2006].

Martínez de Sousa, José (1993), Diccionario de bibliología y ciencias afines. Madrid: Fundación Germán Sánchez Ruipérez, 1993.

Mendoza Díaz-Maroto, Francisco (2002). La pasión por los libros: un acercamiento a la bibliofilia. Madrid: Espasa, 2002. 


\section{6}

Mendoza Díaz-Maroto, Francisco (2004). Introducción a la bibliofilia. Valencia: Club Honrad Haebler, 2004.

Moll, Jaime (1985). La bibliografía en la investigación literaria. // Métodos de estudio de la obra literaria. Madrid: Taurus, 1985.

Moreiro González, José Antonio (1990). Introducción bibliográfica y conceptual al estudio evolutivo de la Documentación. Barcelona: PPU, 1990.

Otlet, Paul (1934). Traité de documentation: le livre sur le livre: théorie et pratique, Bruselas: Mundaneum, 1934. Hay traducción española: Tratado de documentación: el libro sobre el libro: teoría y práctica. Murcia, [Universidad de Murcia], 1996.

Peignot, Gabriel (1802/1804). Dictionnaire raisonné de bibliologie. París: Renouard, 1802/1804. 2 vols.

Pritchard,A. (1969). Statistical Bibliography or Bibliometrics?// Journal of Documentation, 25 (1969), 348-349.

Reyes Gómez, Fermín de los (2006). Manual de Bibliografía. Madrid: Castalia, 2006. [En prensa].

Robinson, A.M. Lewin (1922). Introducción a la bibliografía: guía práctica para trabajos de descripción y compilación. Madrid: Fundación Germán Sánchez Ruipérez, 1922.

Rodríguez Parada, Concepción (2002). La enseñanza de la biblioteconomía en Barcelona: de la Escola a la Facultat de Biblioteconomia i Documentació. // BID, 9 (2002). URL: <http:. //www.ub.es/bid/09rodri2.htm>. [Consulta: sept. 2006].

Romani, V. (2000). Bibliologia: avviamento allo studio del libro tipografico, Milano, S. Bonnard (2000).

Roubakin, N.A. (1922). Introduction à la Psychologie Bibliologique: la Psychologie de la Création des Livres, de leur Distribution et Circulation, de leur Utilisation par les Lecteurs, les Écoles, les Bibliothèques, les Librairies, etc.: Théorie et Pratique. París: J. Povolozky, 1922.

Título de grado en Información y Documentación (2004). Madrid: Agencia Nacional de Evaluación de la Calidad y Acreditación, 2004.

Uzanne, Octave (1896). Dictionnaire bibliophilosophique, typologique, iconophilesque, bibliopègique et bibliotechnique, París: Imprimé pour les Sociétaires de 1'Académie des Beaux Livres, Bibliophiles Contemporains, en l'An de Grâce Bibliomaniaque, 1896. 